\title{
xylUW, two genes at the start of the upper pathway operon of TOL plasmid pWWO, appear to play no essential part in determining its catabolic phenotype
}

\author{
Peter A. Williams, Linda M. Shaw, Christopher W. Pitt $\nmid$ and \\ Milka Vreclf
}

School of Biological

Sciences, University of

Wales, Bangor, Gwynedd

LL57 2UW, UK

\author{
Author for correspondence: Peter A. Williams. Tel: +44 1248 382363. Fax: +44 1248370731. \\ e-mail: P.A.Williams@Bangor.ac.uk
}

The upper pathway operon of the toluene catabolic pathway of TOL plasmid pWWO was shown to carry two open reading frames between the start of transcription and xyIC (encoding benzaldehyde dehydrogenase), the first previously reported gene of the operon. These were designated xylUW: xylU encoded a protein of 131 amino acid residues $\left(M_{r} 14244\right)$ which bore no relationship with any protein in the databases, and $x y / W$ encoded a protein of 348 residues $\left(M_{r} 36992\right)$ which was strongly homologous to other long-chain Zn-containing alcohol dehydrogenases. Extracts of Escherichia coli carrying $x y / U W$ in expression vector pTrc99A contained a novel protein corresponding to XyIW, but no NAD+-dependent dehydrogenase activity against benzyl alcohol, mandelate or benzylamine. A mini-Tn5 transposon carrying the meta pathway operon was constructed and from it two strains of Pseudomonas putida were constructed with the normally plasmid-encoded catabolic operons integrated into the chromosome. Three derivatives of plasmid pKNG101 containing modified xylUW genes were constructed, two of which had frameshifts in $x y / U$ and $x y / W$, respectively, and a third with a deletion from the $3^{\prime}$ end of $x y l U$ into the 5 ' end of $x y / W$. The wild-type genes of the two Pseudomonas strains were substituted by the mutant alleles by reverse genetics. The ability of the constructed mutant strains to utilize the aromatic substrates of the TOL pathway was not significantly affected.

Keywords: TOL plasmid pWW0, $x y l$ genes, toluene/xylene catabolism, Pseudomonas putida strain mt-2

\section{INTRODUCTION}

The TOL plasmids encode the catabolism of toluene and some alkyltoluenes (Williams \& Murray, 1974; Worsey \& Williams, 1975; Kunz \& Chapman, 1981) to benzoate and the alkylbenzoates, which are then further metabolized via catechols and meta (extradiol) ring

\footnotetext{
†Present address: Department of Cell Biology, John Innes Centre, Colney Lane, Norwich NR4 7UH, UK.

¥Present address: Veterinary Faculty, Institute of Anatomy, Gerbiciva 60 , 6100 Ljubljana, Slovenia.

Abbreviations: $A D H$, alcohol dehydrogenase; $B A D H$, benzyl alcohol dehydrogenase; TDH, threonine dehydrogenase.

The GenBank accession number for the sequence reported in this paper is U20269.
}

cleavage to metabolites of the tricarboxylic acid cycle (Murray et al., 1972). The genes are organized in two regulons, the 'upper pathway' operon encoding the enzymes for conversion of the hydrocarbons to benzoates (Harayama et al., 1986, 1989) and the 'meta pathway' operon $x y l X Y Z L T E G F Q K I J H$ (Harayama \& Rekik, 1990) encoding the enzymes for further conversion of the benzoates. Until recently the first operon was reported to consist of the genes $x y l C$ (for benzaldehyde dehydrogenase) (Lebens \& Williams, 1985; Shaw \& Harayama, 1990; Inoue et al., 1995), xylMA (for the two components of xylene monooxygenase) (Suzuki et al., 1991; Shaw \& Harayama, 1995), xylB (for benzyl alcohol dehydrogenase, BADH) (Inouye et al., 1981; Shaw \& Harayama, 1990; Shaw et al., 1993) and $x y l N$, an open reading frame encoding a protein of 
unknown function (Harayama et al., 1989). The $x y l$ $C M A B$ region apparently encodes all the enzymic apparatus necessary for the conversion of the hydrocarbons to benzoates. However, the regulatory signals for expression of the operon and the start of transcription (Inouye et al., 1983, 1984, 1990) were recognized as being considerably (1-2 kbp) upstream of the start codon of $x y l C$ (Lebens \& Williams, 1985) but no function had been assigned to the intervening DNA. In this paper we report its nucleotide sequence and describe experiments designed to determine the role of the open reading frames in the catabolism of toluene and its metabolites.

\section{METHODS}

Bacterial strains and plasmids. Escherichia coli strains were grown in liquid culture on Luria broth (LB) and on plates of Isosensitest agar to which antibiotics were added, as appropriate, at the following concentrations $\left(\mu \mathrm{g} \mathrm{ml}^{-1}\right)$ : ampicillin (100), kanamycin (75). pUC plasmids were maintained in $E$. coli strain NM522. Expression plasmid pTrc99A was purchased from Pharmacia. Pseudomonas strains were all derivatives of $P$. putida mt-2, the original host of TOL plasmid pWW0, and were grown on minimal salts medium with carbon sources added at $5 \mathrm{mM}$ for aromatics and $10 \mathrm{mM}$ for succinate. Growth on toluene, $m$-xylene and $p$-xylene was assessed on minimal medium agar plates with the liquid hydrocarbon presented as vapour from small glass tubes in the inverted lid of the Petri dish.

Transfer of plasmids to $P$. putida from $E$. coli. Derivatives of pUT and pKNG101 plasmids were transformed into $E$. coli

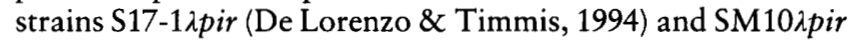
(Miller \& Mekalanos, 1988) as respective donors and into CC118גpir (Herrero et al., 1990) for maintenance. Donor and recipient cultures were grown overnight in $5 \mathrm{ml} \mathrm{LB}$ with appropriate antibiotic. Then $1 \mathrm{ml}$ of each was harvested, resuspended in $1 \mathrm{ml}$ sterile minimal medium and $10 \mu \mathrm{l}$ of each was mixed on an LB agar plate and incubated overnight. The resultant patch of cells was resuspended in $1 \mathrm{ml}$ sterile minimal medium and $100 \mu \mathrm{l}$ was spread on to appropriate selection medium for single colonies of transconjugants.

Insertion of the meta pathway into chromosome of $\boldsymbol{P}$. putida. The $17.5 \mathrm{kbp}$ HindIII insert (HA) was cut from plasmid pWW53-3301 (Keil et al., 1987) with HindIII and inserted into the HindIII site of pUC18Sfi (Herrero et al., 1990) to form pUC18Sfi(HA). HA carries the complete meta pathway operon plus its positive regulator gene $x y l S$ from TOL plasmid pWW53 (Keil et al., 1987). Since HA has four internal Sfil sites it was necessary to remove the insert with AvrII which cuts the two Sfil sites on the pUC18Sfi plasmid but does not cut within the HA fragment. Plasmid pUTKm2 is a mini-Tn5 carrier plasmid (De Lorenzo et al., 1990; De Lorenzo \& Timmis, 1994) in which the kanamycin resistance gene has been inserted into the mini-transposon as an Sfil fragment and is excisable with AvrII. Plasmids pUC18Sfi(HA) and pUTKm2 were both digested with AvrII and the mixture ligated to form pUTHA, in which HA was inserted within the mini-Tn 5 transposon in place of the kanamycin resistance gene. This plasmid was transformed into $E$. coli CC118גpir and, for delivery to $P$.

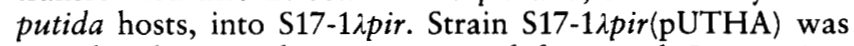
mated with $P$. putida KT2442 $\Delta$ (a gift from V. de Lorenzo) as recipient, selecting for growth on $m$-toluate minimal medium: KT $2442 \Delta$ has a mini-Tn5 insertion in its chromosome which carries both the $x y l U W C M A B N$ operon and its positive regulator gene $x y l R$. A single transconjugant, $\mathrm{PaW} 140$, was retained. With its chromosomal insertion of the TOL upper pathway operon, strain KT2442 $\Delta$ was only able to grow on toluene, benzyl alcohol, benzaldehyde and benzoate, which are further metabolized by the chromosomal $\beta$-ketoadipate pathway. However, PaW140, with the additional complete meta pathway operon in its chromosome, was additionally able to grow on the 3-methyl and 4-methyl analogues, which can be metabolized via the meta pathway.

Construction of mutations within the $x y / U W$ region. Plasmid pWW0-7000 (Fig. 1) was cut at its unique XhoI site within $x y l U$ and the recessed $3^{\prime}$ ends were filled in with Klenow enzyme to produce blunt ends which were religated to obtain plasmid pWW0-7010 (Fig. 1) carrying the mutated gene designated $x y l U^{*}$. Plasmid pWW0-7001 was made by insertion of the $2.05 \mathrm{kbp} \mathrm{SmaI-BamHI}$ fragment from pWW0-7000 into expression vector $\mathrm{pTrc} 99 \mathrm{~A}$ cut with EcoICR 1 (at its $\mathrm{SacI}$ site) and $B a m H I$. The single ClaI site in $x y l W$ on plasmid pWW07001 was removed by cutting with ClaI, filling in with Klenow and religating to produce $\mathrm{pWW} 0-7011$ carrying the mutated allele $x y l W^{*}$ (Fig. 1). $x y l W^{*}$ was then removed from $\mathrm{pWW0}$ 7011 on a $2.6 \mathrm{kbp}$ BstEII-Bam HI fragment and cloned into the equivalent BstEII-BamHI sites on $\mathrm{pWW}-7000$ to generate pWW0-7012 (Fig. 1). Both pWW0-7011 and pWW0-7012 were cut with $S m a \mathrm{I}$ and $B a m \mathrm{HI}$ and the $2.8 \mathrm{kbp}$ fragments from each were separately ligated into the SmaI-BamHI sites of pKNG101, producing plasmids pWW0-7020 and pWW0-7021 respectively (Fig. 1 ).

A third mutation was constructed by deletion between the PstI sites at 1.75 and $2.5 \mathrm{kbp}$, removing the $3^{\prime}$ end of $x y l U$ and the $5^{\prime}$ end of $x y l W$. This could not be constructed directly from pWW0-7000 since a third PstI site is present on the multicloning site of the vector. To circumvent this problem, pUC18 (Vieira \& Messing, 1982) was first modified by removing its PstI site by digestion with PstI, filling in the $3^{\prime}$ ends with Klenow enzyme and religating to form pUC18NP. The $3.9 \mathrm{kbp}$ KpnI-BamHI fragment from pWW0-7000 was then cloned into the KpnI and BamHI sites of pUC18NP to form plasmid pWW0-7013 (not shown). This was digested with PstI and

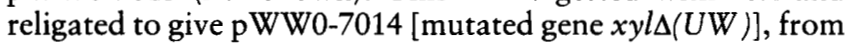
which the SmaI-BamHI fragment was removed and ligated into pKNG101 to form the suicide delivery plasmid pWW07022 (Fig. 1).

Reverse genetics with pKNG101 derivatives. The broad-hostrange suicide vector pKNG101, designed for reverse genetics in Gram-negative bacteria (Kaniga et al., 1991), was used to incorporate the $x y l U W$ mutations described above into the appropriate Pseudomonas strains. As in the protocol of Kaniga et al. (1991), sucrose-resistant (Suc ${ }^{r}$ ) revertants were selected from sucrose-sensitive (Suc $\left.{ }^{\mathrm{s}}\right)$, streptomycin-resistant $\left(\mathrm{Str}^{\mathrm{r}}\right)$ transconjugants in which the complete plasmid had integrated into the chromosome by a single homologous recombination at the wild-type $x y l U W$ locus. A proportion of these revertants should have the wild-type gene replaced by the mutated gene.

DNA manipulations and electrophoretic analysis of recombinant plasmids. Plasmid DNA was prepared from E. coli hosts using commercial Qiagen or Pharmacia plasmid preparation kits. Plasmid DNA from pWW0 was prepared from $P$. putida by the sucrose density method (Wheatcroft \& Williams, 1981). Digestion of DNA with restriction enzymes, ligations, preparation of competent cells, transformations, agarose gel electrophoresis of digested plasmid DNA and PCR were carried out according to the suppliers' instructions and/or by standard procedures. 


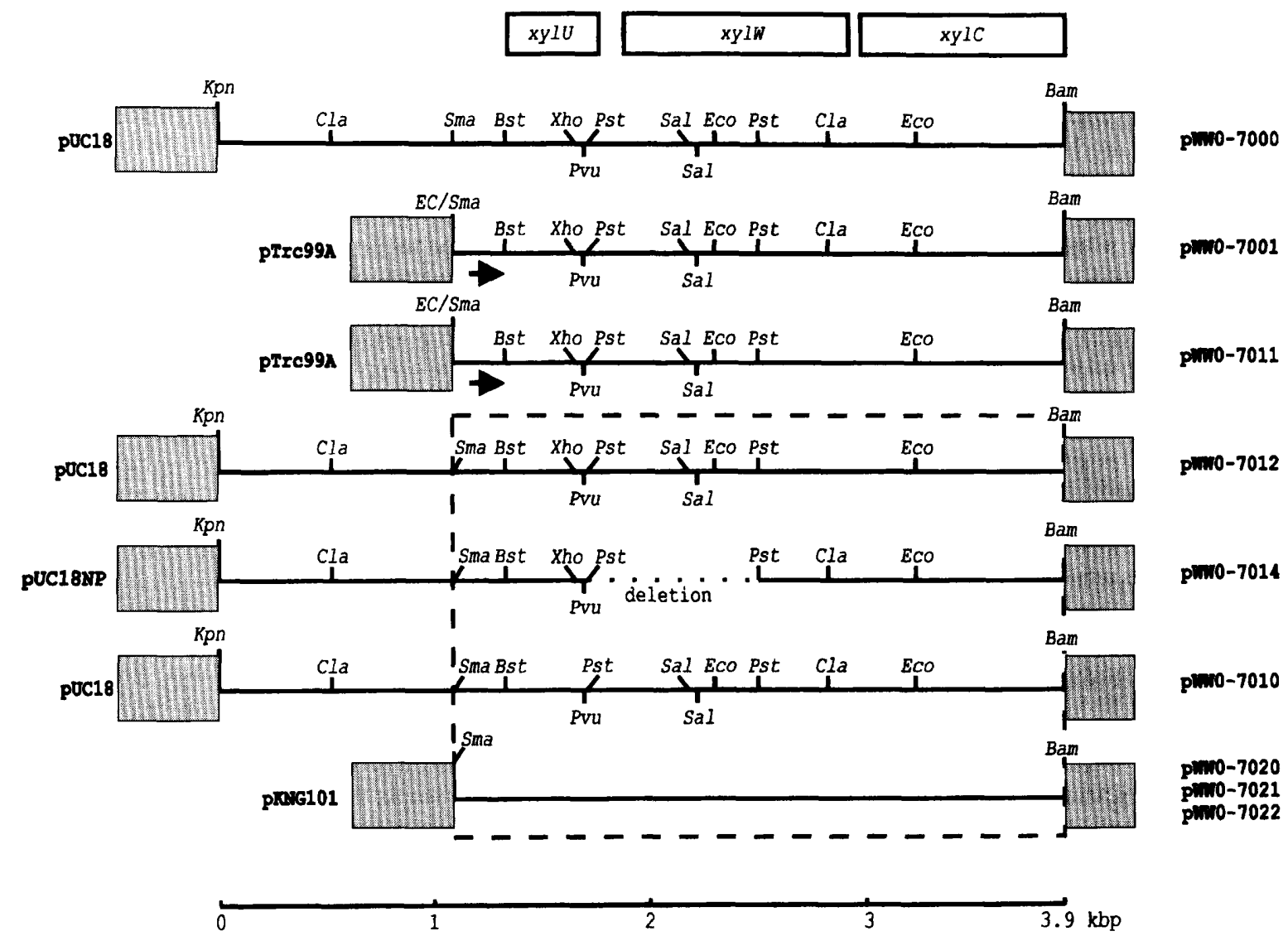

Fig. 1. Restriction maps of recombinant plasmids derived from the $5^{\prime}$ end of the upper pathway operon of TOL plasmid pWW0: the plasmid designations are on the right. Plasmids pWW0-7012 and -7020 carry the $x y / W^{*}$ allele, pWW0-7014 and -7021 the $x y l \Delta(U W)$ allele, and pWW0-7010 and -7022 the $x y l U^{*}$ allele. The grey boxes denote the termini of the vectors, which are named on the left, and the solid lines represent the inserts of pWWO DNA. The restriction sites from the multicloning sites of each vector into which the pWWO DNA is inserted are above the ends of each terminus. The direction of transcription from the promoter of expression vector pTrc99A in pWW0-7001 and pWW0-7011 is denoted by the arrows. The box surrounded by dashed lines spanning plasmids pWW0-7012, -7014 and -7010 delineates the three Smal-BamHI fragments cloned into pKNG101 to form pWW0-7020, -7021 and -7022 respectively. Abbreviations for restriction sites: Bam, BamHI; Bst, BstEll; Cla, Clal; Eco, EcoRl; EC/Sma, the hybrid site formed from blunt-end ligation of a Smal site on the insert into an EcolCR1 site on the vector; Kpn, Kpnl; Pst, Pstl; Sal, Sall; Pvu, Pvull; Xho, Xhol. Note that the Pstl site normally to the right of the BamHI multicloning site in pUC18 is absent in pWW0-7014, having been eliminated in the formation of pUC18NP.

Nucleotide sequence determination. Sequence determination was performed on dsDNA cloned in pUC plasmids using Pharmacia sequencing kits. The starting plasmid used for the sequence determination was the pUC19-based pWW0-3016, which has HindIII fragment HD $(10 \mathrm{kbp})$ from pWW0 as insert. A $3.9 \mathrm{kbp} \mathrm{KpnI-BamHI}$ subclone of pWW0-3016 was inserted into pUC19 to form pWW0-7000, which was known to carry the region previously unsequenced from the promoter region $\mathrm{Pu}$ past the $5^{\prime}$ end of $x y l C$. Initially primers were designed using the published sequences of the promoter $\mathrm{Pu}$ (Inouye et al., 1984, 1990) and xylC (Shaw et al., 1993) and subsequently two series of nested deletions were made from each end using the Exonuclease III kit (Pharmacia): gaps without overlaps and regions of ambiguity at the ends of sequences were sequenced using synthetic oligonucleotide primers. The sequences of both strands were determined over a region of 1734 bp overlapping the published $\mathrm{Pu}$ and $x y l C$ sequences.

DNA and protein sequence analysis. These were performed using the modules of DNASTAR. Comparison of nucleotide and protein sequences with the databases was carried out using the BLAST programs.

\section{RESULTS}

\section{Analysis of nucleotide sequence}

Two open reading frames were identified between the promoter region $\mathrm{Pu}$ and the start codon of $x y l C$ for benzaldehyde dehydrogenase (Fig. 2). The ATG start codon of the first, designated $x y l U$, was originally shown to be the translational start of the operon but was wrongly identified as the reading frame for xylene monooxygenase (Inouye et al., 1984). The structural genes for all the enzymic steps of the conversion of toluene to benzoate $(x y l C M A B)$ were subsequently shown to be $>1 \mathrm{kbp}$ downstream of this codon (Lebens \& Williams, 1985; Harayama et al., 1986). The ORF designated $x y l U$ appears to encode a small protein of 


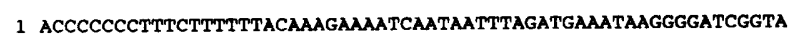
61 TAAGCAATGGCATGGCGGTTGCTAGCTATACGAGACTTAAAATAAAAATAGTGGTGACCC

UWI $* * * * * * * * * * * * * * * * * * \rightarrow$
121 TTCAATGTTGTATTTTCTCAACTCTGTTCAGATTGGTTCTMTCGCATGTATATCCTCA 1 TTCAATGTTGTATTTTCTCAACTCTGTTCAGATTGGTTGCTTTCGCCATGTATATCCTCA
XY1U: $M$ 181 AAGCGGGCCAGCCGTAGCCGTTACGCCTGGAGTCTGTGACGCTGGCCTAGTGCTTTCTGA

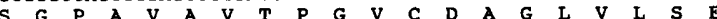
241 GGGCGAGCCCAAGCAGGCTAGCCCGACCACCAATTTTGGTGAGGCCAAGGTCATTGGCGT

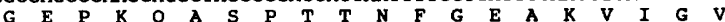

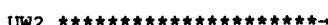
301 GCGTGCGACTTTGCGCACCATTTTCGTCGCAGTTATCGGCTTCATCTCTCGTGATAGGG 361 TTATAGATGCCAGTCCAACGGTGCGGCTGCATCAATCTTCAATCTCATCGCTAACCTCAA 421 CCGACTATATGCCCAGGTCTGCAACGATATATCCAGCCTGTCGCGCATrCGCTCGAGCCT $\begin{array}{llllllllllllllllllllllll}R & L & Y & A & Q & V & C & N & D & I & S & S & L & S & R & I & R & S & S & L\end{array}$ PVuIIPstI

481 TAGTCATCTACCGCAGTCACAGCTGCAGCGACATTACTAACTAGTCGTGTCGATCTCGGA

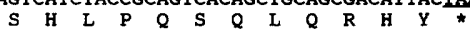

541 AGCTGTGCGTTTGTTGCCGAGAACTTGGCTGCATGTGATGCATTAAGTGATTTGATATCG 601 CCGTCCTTGCTACCTATAACATTTGACGTCTACATAGGTGATTCAATGCTCGCGATAAGG Xylw: $M$ L A I $R$

661 AAAACTAAAGCAGGGCCAGGAGGGCTATCTGTCGATGATGTGGCCGCCCCTAAAGAGGCC $\begin{array}{llllllllllllllllllll}K & \text { T } & K & \text { A } & G & \text { P } & G & G & \text { L } & \text { S } & \text { V } & \text { D } & \text { D } & \text { V } & \text { A } & \text { A } & \text { P } & \text { K } & \text { E } & \text { A }\end{array}$ 721 CACAGTACTGACGTGCTGGTGAAAGTTGAAGGGCCGGTATCTGTGGTACTGACTTGTTG

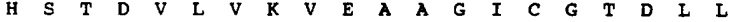
781 ATCTATAAATGGGGCGAGTTCGCCAAAAGAATGAAACTCCCCACTATCTTGGGTCATGAA 841 GTTAGCGGGGTGATTGAACAGGTCGGATCTGACGTTAAAGGTCTAAGGCCTGGGATGCGC $\begin{array}{lllllllllllllllllllll}V & S & G & V & I & E & Q & \text { V } & G & S & D & V & K & G & \text { L } & R & \text { P } & G & M & R\end{array}$ 901 GTCAGTCTGGAGAGTCATCTCCCTTGTGGAACGTGCTACACGTGTCGACGCGGTTGGGCG $\begin{array}{llllllllllllllllllll}V & S & L & E & S & H & L & P & C & G & T & C & Y & T & C & R & R & G & W & A\end{array}$ 961 CATGTTTGTCCTAAGACGCGGTATCCGGGGGTCGACTTTGATGGAGGCTTCGCGTCTTTC

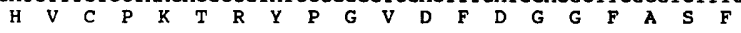
1021 GTTGTGGTTCCGGAAAGCGTGTGTTGGCCGGTGCCGTGCGGAATTCCTCCGCTCCAGGCG $\begin{array}{lllllllllllllllllllllllll}V & V & V & P & E & S & V & C & W & P & V & P & C & G & I & P & P & L & Q & A\end{array}$ 1081 ACAATGATGGAGCCGTTCGGGCTTGCGGTGCATGCAAGTCTGGAGGGTTCTGGGGTTCG $\begin{array}{llllllllllllllllllll}T & M & M & E & P & F & G & L & A & V & H & A & S & I & E & G & S & G & V & S\end{array}$ 1141 GGATTGAATGTTCTTGTTTCTGGTTGCGGTCCGATCGGACTTATGAACATTGCAGCCGCC $\begin{array}{llllllllllllllllllll}G & L & N & V & L & V & S & G & C & G & P & I & G & L & M & N & I & A & A & A\end{array}$

1201 AAAGCACTGGGAGCAAGCAAGGTCATTGCAACGGACATTCATCCTCTGCGCCTCACTGCA $\begin{array}{llllllllllllllllllll}\mathbf{K} & \mathbf{A} & \mathrm{L} & \mathbf{G} & \mathbf{A} & \mathbf{S} & \mathbf{K} & \mathbf{V} & \mathbf{I} & \mathbf{A} & \mathbf{T} & \mathbf{D} & \mathbf{I} & \mathbf{H} & \mathbf{P} & \mathbf{L} & \mathbf{R} & \mathbf{L} & \mathbf{T} & \mathbf{A}\end{array}$

1261 GCAGCCAAAATGGGGGCGGATGAATGCATTAATGCAACTGAAGAGAGCGTTTATAAGACT A A K M G A D E C I N A T E E S V Y 1321 GTGCGTTCGCTGCTCGGTGAGAGGGGAGTAGATGTTGCAATTGACTATTCTGGGCAAGCA

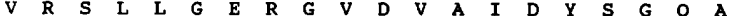
1381 GCGGCGTTAAAGGAGCTTATTCAATCCATCACCCATGGTGGTGAGTTGCGACTGATGGGC $\begin{array}{llllllllllllllllllll}\text { A } & A & L & \text { K } & \text { E } & \text { L } & \text { I } & \mathbf{Q} & \text { S } & \text { I } & \text { T } & \text { H } & \text { G } & \text { G } & \text { E } & \text { L } & \text { R } & \text { L } & \text { M } & G\end{array}$ 1441 GTCCCGTCACATGATATTTTGCTCAACTTAGAGGAGTGGCTTTTCAAAGGCCTTATTGTA 1501 CGTGGCCTGCATGGCAGGCGTCTGTATGAAACATGGGAGCGTTCAACTAGCTTATTGGTT

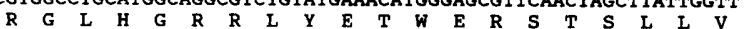
1561 ACAGGGCGTGTTGATTTGTCTTCGCTCGTATCGCATCGATTGCCACTATCGGCGGCAGAG $\begin{array}{llllllllllllllllllll}T & G & R & V & D & L & S & S & L & V & S & H & R & L & P & L & S & A & A & E\end{array}$ 1621 GATGCGTTCGAAATGGCCCTAAATGGTCAGTCCTTGAAAATCCTGTTTGAGCCCAATGGC $\begin{array}{lllllllllllllllllll}D & A & F & E & M & A & L & N & G & Q & S & L & K & I & L & F & E & P & N\end{array}$

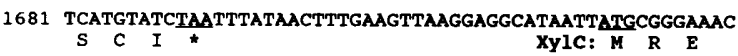

Fig. 2. Nucleotide sequence of the $5^{\prime}$ end of the upper pathway operon of TOL plasmid pWWO and the predicted amino acid sequences of XylU and XylW. Relevant restriction sites (see Fig. 1) are overlined above the nucleotide sequence and the DNA sequences corresponding to the two oligonucleotide primers for the 5' ends of the two sets of forward PCR primers (UW1 and UW2) are denoted by the asterisks above the sequence. The reverse PCR primer (UW3) starts in xy/C beyond the end of the DNA sequence determined in this study but overlaps into its 3 end: its nucleotide sequence is presented in Results.
131 amino acid residues $\left(M_{\mathrm{r}} 14244\right)$ with no pronounced hydrophobic character. It shared significant homology with only one protein in the databanks, TmbU (GenBank U41301). Downstream of $x y l U$ was $x y l W$, a second ORF of 348 codons $\left(M_{r} 36992\right)$. This terminated just upstream of the start of $x y l C$ and shows significant homology with the family of group 1 long-chain $\mathrm{Zn}$ containing alcohol dehydrogenases (ADHs). Apart from TmbW (see Discussion) the two proteins most similar to it are the 341-residue threonine dehydrogenase (TDH) from E. coli (GenBank P07913) and the sorbitol dehydrogenase from Bacillus subtilis (GenBank Q06004). Alignment of the amino acid sequences of XylW and TDH introduced only five gaps of a single residue, and showed $37 \%$ identity and $62.9 \%$ similarity. XylW had only limited homology (28\% identity and $53 \%$ similarity) with benzyl alcohol dehydrogenase (BADH; XylB), another Group 1 ADH (Shaw et al., 1993), but negligible homology with benzaldehyde dehydrogenase (XylC), the adjacent downstream gene product (Inoue $e t$ al., 1995).

The $x y l U W$ region was cloned into vector $\mathrm{p} \operatorname{Trc} 99 \mathrm{~A}$ to form plasmid pWW0-7001 for expression of XylU and XylW (Fig. 1). PAGE gels were run of the SDS-treated extracts of E. coli JM105 carrying pWW0-7001 with and without induction by IPTG. A unique protein band of apparent $M_{\mathrm{r}} \sim 38000$, corresponding to XylW, was observed but no band was detected which could be identified as XylU (data not shown).

\section{Formation of mutated $P$. putida strains and their authentication by PCR}

E. coli SM10גpir strains carrying the three plasmids pWW0-7020 (xylU*), pWW0-7021 (xylW*) and pWW0$7022[x y l \Delta(U W)]$ were separately mated with $P$. putida strains KT2442 $\Delta$ and $\mathrm{PaW} 140$ and transconjugants were selected on succinate $/ \mathrm{Km}$ plates. Four transconjugants were retained in each case and their phenotypes checked: in all cases they were $\mathrm{Km}^{\mathrm{r}}$ and grew on the same range of aromatic substrates as their parent strains but failed to grow on succinate $/ \mathrm{Km}$ plates containing $5 \%(\mathrm{w} / \mathrm{v})$ sucrose. Cultures of all transconjugants were grown in LB overnight and plated on to succinate plates containing $5 \%$ sucrose at a density of $10^{7}-10^{8}$ cells per plate. Single colonies appeared and 20 from each were retained and shown to be $\mathrm{Str}^{\mathrm{s}} \mathrm{Suc}^{\mathrm{r}}$, as expected (Kaniga et al., 1991).

The relevant genotype of the strains was determined by PCR. Two forward 19mer primers (UW1 and UW2) were designed from the $5^{\prime}$ end of $x y l U$ (see Fig. 1) and a single 20mer reverse primer (UW3; 5' AATACGCCCTTTGTTTTCTCG $3^{\prime}$ ) spanning the start codon of xylC (Shaw et al., 1993): these were chosen such that the PCR products, uncut and cut with restriction enzymes, from mutant alleles of $x y l U$ and $x y l W$ could be distinguished from the wild-type alleles of the genes using both forward-reverse primer combinations. Genomic DNA from each Pseudomonas transconjugant and 
Table 1. Phenotypes of mutant $P$. putida strains

\begin{tabular}{|c|c|c|c|c|c|}
\hline $\begin{array}{l}\text { Parent } P \cdot \text { putida } \\
\text { (genotype) }\end{array}$ & $\begin{array}{l}\text { Integrated } \\
\text { plasmid }\end{array}$ & $\begin{array}{c}\text { Transconjugant } \\
\text { straint }\end{array}$ & Revertants & $\begin{array}{l}\text { Revertant } \\
\text { genotype }\end{array}$ & $\begin{array}{l}\text { Revertant } \\
\text { phenotype }\end{array}$ \\
\hline $\begin{array}{l}\text { KT2442A } \\
\quad(x y l U W C M A B N x y l R)\end{array}$ & $\begin{array}{l}\mathrm{pWW} 0-7020 \\
\mathrm{pWW} 0-7021 \\
\mathrm{pWW} 0-7022\end{array}$ & $\begin{array}{l}\text { PaW7041 } \\
\text { PaW7054 }\end{array}$ & $\begin{array}{l}\text { PaW7032R1 } \\
\text { PaW7032R3 } \\
\text { PaW7041R2 } \\
\text { PaW7041R3 } \\
\text { PaW7054R1 } \\
\text { PaW7054R6 }\end{array}$ & $\begin{array}{l}x y l U^{*} x y l W \\
x y l U x y l W \\
x y l U x y l W * \\
x y l U x y l W \\
x y l U x y l W \\
x y l \Delta(U W)\end{array}$ & $\mathrm{Str}^{\mathrm{s}} \mathrm{Suc}^{\mathrm{r}} \mathrm{Tln}^{+} \mathrm{Bal}^{+} \mathrm{Bza}^{+} \mathrm{Ben}^{+}$ \\
\hline $\begin{array}{l}\mathrm{PaW} 140 \\
(x y l U W C M A B N x y l R \\
x y l X Y Z L T E G F J Q K I H \quad x y l S 1\end{array}$ & $\begin{array}{l}\text { pWW0-7020 } \\
\text { pWW0-7021 } \\
\text { pWW0-7022 }\end{array}$ & $\begin{array}{l}\text { PaW7037 } \\
\text { PaW7045 } \\
\text { PaW7055 }\end{array}$ & $\begin{array}{l}\text { PaW7037R4 } \\
\text { PaW7037R5 } \\
\text { PaW7045R1 } \\
\text { PaW7045R2 } \\
\text { PaW7055R1 } \\
\text { PaW7055R2 }\end{array}$ & $\begin{array}{l}x y l U^{*} x y l W \\
x y l U x y l W \\
x y l U x y l W \\
x y l U x y l W * \\
x y l U x y l W \\
x y l \Delta(U W)\end{array}$ & $\begin{array}{l}\mathrm{Str}^{8} \mathrm{Suc}^{\mathrm{r}} \mathrm{Tln}^{+} \mathrm{Mxy}^{+} \mathrm{Pxy}^{+} \\
\mathrm{Bal}^{+} 3 \mathrm{MBal}^{+} 4 \mathrm{MBal}^{+} \mathrm{Bza}^{+} \\
3 \mathrm{MBza}^{+} 4 \mathrm{MBza}^{+} \mathrm{Ben}^{+} \\
\mathrm{Mtol}^{+} \mathrm{Ptol}^{+}\end{array}$ \\
\hline
\end{tabular}

† All transconjugants were $\operatorname{Str}^{r} \operatorname{Suc}^{\mathrm{s}}$ and carried both a wild-type and a mutant allele of the relevant gene.

$\ddagger$ Phenotype designations: the ability to use the following as sole carbon source. Tln, toluene; Mxy, $m$-xylene, Pxy, $p$-xylene; Bal, benzyl alcohol; 3MBal, 3-methylbenzyl alcohol; 4MBal, 4-methylbenzyl alcohol; Bza, benzaldehyde; 3MBza, 3-methylbenzaldehyde; 4MBza, 4methylbenzaldehyde; Ben, benzoate; Mtol, $m$-toluate (3-methylbenzoate); Ptol, $p$-toluate (4-methylbenzoate).

its Suc revertants was subjected to PCR and the amplified fragment was subjected to digestion with the appropriate restriction enzymes. The initial transconjugants $\left(\operatorname{Str}^{\mathrm{r}} \mathrm{Suc}^{\mathrm{s}}\right)$ with the chromosomally integrated plasmid should have contained both a wild-type and a mutant gene: a single transconjugant from each mating was retained which produced two amplified fragments with the expected patterns of digestion. The $\operatorname{Str}^{\mathrm{s}} \mathrm{Suc}^{\mathrm{r}}$ revertants from each of these transconjugants were examined in the same way until one with a mutant gene and one with the wild-type gene were identified: one of each was retained. In no case was it necessary to examine more than eight revertants of any transconjugant before one unambiguous example of each kind was found.

All of the strains, with mutant or with wild-type genes, derived from $P$. putida KT2442 $\Delta$ grew on toluene, benzyl alcohol, benzaldehyde and benzoate, and all those derived from PaW140 grew on the same four substrates as well as their 3-methyl and 4-methyl analogues (Table 1) although growth on the 4-methylated substrates of all strains was considerably slower than on the 3-methyl and unsubstituted substrates. No differences were apparent in the rates of growth on plates between any parent strain and its derivatives and no significant differences were observed in their exponential growth rates in liquid cultures.

\section{DISCUSSION}

The results presented show the presence of two new genes $(x y l U W)$ at the beginning of the upper pathway operon of the TOL plasmid. XylU joins a number of other putative ORFs found in aromatic catabolism which appear to have no counterparts in the databanks, for which functions have not yet been determined and which do not appear to be related to each other. These include XylN at the opposite end of the same operon, TodR on the alternative tod pathway for toluene utilization (Wang et al., 1995), ORFs in biphenylutilizing strains LB400 (Erickson \& Mondello, 1992) and Pseudomonas pseudoalcaligenes KF707 (Taira et al., 1992), and TbuU from the toluene-3-monoxygenase pathway of P. pickettii (Byrne et al., 1995). There is one other ORF highly homologous to XylU in the databanks, TmbU. However, this is encoded by a chromosomal sequence carrying genes for trimethylbenzene catabolism (Polissi et al., 1990) which includes other genes also highly homologous to the $\mathrm{pWW} 0 x y$ lgene products: downstream of $x y l U$ is $t m b W(=x y l W)$ and a partial sequence for $t m b C(=x y l C)$, and upstream are $t m b R$ $(=x y l R)$ and $t m b S(=x y l S):$ undoubtedly this DNA is another $\mathrm{pWW} 0$-like sequence, but it has a different gene order and appears to have become chromosomally located.

However, it is the possible function of XylW which is arguably the most intriguing of the two new TOL ORFs. Its sequence indicates strongly that it is an ADH. Apart from its virtual identity (343/348 residues identical) with $\mathrm{TmbW}$, it has greatest similarity to TDH from $E$. coli (Epperly \& Dekker, 1991; Chen et al., 1995) and even shares a limited homology with BADH (XylB) from the same operon, which is also a Group $1 \mathrm{Zn}-\mathrm{ADH}$ (Shaw et al., 1993). XylW has many of the characters found in microbial ADHs (Reid \& Fewson, 1994). Primarily it has the sequence GHEVSGVIEQVGSDV (residues 63-77) corresponding to the typical $\mathrm{Zn}-\mathrm{ADH}$ signature GHExxGxxxxxGxxV and containing the His which binds to the zinc ion. Other residues shared with microbial ADHs are the sequence Cys-His-Ser-Asp 
(residues 40-43), corresponding to the consensus of CysHis-Thr-Asp (Reid \& Fewson, 1994); His-64, Glu-65, Ala-185, corresponding to the archetypal horse liver $\mathrm{ADH}(\mathrm{HLADH})$ residues 67, 68, 211 respectively; and Asp-197, found in all NAD $(\mathrm{H})$-dependent enzymes but replaced by Gly in the NADP $(\mathrm{H})$-dependent enzymes (Reid \& Fewson, 1994). An unusual feature which XylW shares with two BADHs, XylB and BADHII from Acinetobacter calcoaceticus (D. Gilooly \& C. A. Fewson, personal communication), is the replacement of a conserved His (corresponding to HLADH-51) by a hydrophobic amino acid (Leu, $\mathrm{Val}$ and Ile respectively).

The incorporation of mutant alleles of $x y l U$ and $x y l W$ into a chromosomally integrated copy of the $x y l$ $U W C M A B N$ operon produces no apparent change in the catabolic phenotype of the host strains and the mutants grow as well as wild-types on all nine of the upper pathway substrates tested. It is possible that the single restriction sites modified in two of the constructions, the XhoI site in $x y l U^{*}$ and the ClaI site in $x y l W^{*}$, are both close enough to the $3^{\prime}$ ends of the respective genes for the two modified gene products to retain activity. This cannot be true for the third mutation, the PstI deletion producing $x y l \Delta(U W)$, as it removed the start codon of $x y l \mathrm{~W}$ and $>50 \%$ of the $5^{\prime}$ end of the gene and fused it to the $5^{\prime}$ end of $x y l U$.

Two roles for XylW seem possible which do not conflict with these findings. One is that it functions as a second $\mathrm{BADH}$, the action of which merely supplements that of $\mathrm{XylB}$ so that its loss is not a limiting factor in growth. From preliminary results (not shown) we think that this is unlikely since we have been unable to detect any such activity from an expression vector using the standard assay procedure for XylB (Worsey \& Williams, 1975). This region is also highly conserved in a number of other TOL plasmids, as seen by comparison of the restriction maps of this operon (Assinder \& Williams, 1990). This implies that there is a strong pressure for its retention and that it is not simply a superfluous duplication. A second possibility is that XylW is part of a convergent branch of the pathway which converts some growth substrate, not yet identified, into one of the other recognized TOL pathway metabolites. In preliminary experiments we have tried only two which might fill this role and be converted to benzaldehyde, mandelic acid and benzylamine. Neither served as substrates for XylW in a preliminary dehydrogenase assay procedure using extracts of $E$. coli carrying pWW0-7001, nor did they serve as growth substrates for strains with a functional $x y l W$ gene as compared with strains with a mutated or absent $x y l W$.

\section{ACKNOWLEDGEMENTS}

This paper is publication 14 from the Centre for Biological Transformation in the Yale Institute for Biospheric Studies. We should like to express our thanks to Victor De Lorenzo for strains and for several early discussions and crucial suggestions; to Guy Cornelis for pKNG101; to L. Nicholas Ornston and his colleagues in the Department of Biology at Yale for a user-friendly environment in which part of the work was carried out whilst on sabbatical leave; and to Adam R. Johnson for electronic communications about TDH.

\section{REFERENCES}

Assinder, S. J. \& Williams, P. A. (1990). The TOL plasmids determinants of the catabolism of toluene and the xylenes. Adv Microb Physiol 31, 1-69.

Byrne, A. M., Kukor, J. J. \& Olsen, R. H. (1995). Sequence analysis of the gene cluster encoding toluene-3-monooxygenase from Pseudomonas pickettii PKO1. Gene 154, 65-70.

Chen, Y. W., Dekker, E. E. \& Somerville, R. L. (1995). Functional analysis of Escherichia coli threonine dehydrogenase by means of mutant isolation and characterization. Biochim Biophys Acta 1253, 208-214.

De Lorenzo, V. \& Timmis, K. N. (1994). Analysis and construction of stable phenotypes in Gram-negative bacteria with Tn5-derived and Tn10-derived minitransposons. Methods Enzymol 235, 386-405.

De Lorenzo, V., Herrero, M., Jakubzik, U. \& Timmis, K. N. (1990). Mini-Tn5 transposon derivatives for insertion mutagenesis, promoter probing, and chromosomal insertion of cloned DNA in Gram-negative eubacteria. J Bacteriol 172, 6568-6572.

Epperly, B. R. \& Dekker, E. E. (1991). L-Threonine dehydrogenase from Escherichia coli-identification of an active-site cysteine residue and metal-ion studies. J Biol Chem 266, 6086-6092.

Erickson, B. D. \& Mondello, F. J. (1992). Nucleotide sequencing and transcriptional mapping of the genes encoding biphenyl dioxygenase, a multi-component polychlorinated biphenyldegrading enzyme in Pseudomonas strain LB400.J Bacteriol 174, 2903-2912.

Harayama, S. \& Rekik, M. (1990). The meta-cleavage operon of TOL degradative plasmid pWW0 comprises 13 genes. Mol Gen Genet 221, 113-120.

Harayama, S., Leppik, R. A., Rekik, M., Mermod, N., Lehrbach, P. R., Reineke, W. \& Timmis, K. N. (1986). Gene order of the TOL catabolic plasmid upper pathway operon and oxidation of both toluene and benzyl alcohol by the $x y l A$ product. J Bacteriol 167 , 455-461.

Harayama, S., Rekik, M., Wubbolts, M., Rose, K., Leppik, R. A. \& Timmis, K. N. (1989). Characterization of five genes in the upper pathway operon of TOL plasmid pWW0 from Pseudomonas putida and identification of the gene products. J Bacteriol 171, 5048-5055.

Herrero, M., de Lorenzo, V. \& Timmis, K. N. (1990). Transposon vectors containing non-antibiotic markers for cloning and stable chromosomal insertion of foreign genes in Gram-negative bacteria. J Bacteriol 172, 6557-6567.

Inoue, J., Shaw, J. P., Rekik, M. \& Harayama, S. (1995). Overlapping substrate specificities of benzaldehyde dehydrogenase (the $x y l C$ gene product) and 2-hydroxymuconic semialdehyde dehydrogenase (the $x y l G$ gene product) encoded by TOL plasmid pWW0 of Pseudomonas putida. J Bacteriol 177, 1196-1201.

Inouye, S., Nakazawa, A. \& Nakazawa, T. (1981). Molecular cloning of TOL gene $x y l B$ and gene $x y l E$ in Escherichia coli. $J$ Bacteriol 145, 1137-1143.

Inouye, S., Nakazawa, A. \& Nakazawa, T. (1983). Molecular cloning of regulatory gene $x y l R$ and operator-promoter regions of the $x y l A B C$ and $x y l D E G F$ operons of the TOL plasmid. $J$ Bacteriol 155, 1192-1199.

Inouye, S., Ebina, Y., Nakazawa, A. \& Nakazawa, T. (1984). Nucleotide sequence surrounding transcription initiation site of 
$x y l A B C$ operon on TOL plasmid of Pseudomonas putida. Proc Natl Acad Sci USA 81, 1688-1691.

Inouye, S., Gomada, M., Sangodkar, U. M. X., Nakazawa, A. \& Nakazawa, T. (1990). Upstream regulatory sequence for transcriptional activator $x y l R$ in the first operon of xylene metabolism on the TOL plasmid. $J$ Mol Biol 216, 251-260.

Kaniga, K., Delor, I. \& Cornelis, G. R. (1991). A wide host-range suicide vector for improving reverse genetics in Gram-negative bacteria - inactivation of the blaA gene of Yersinia enterocolitica. Gene 109, 137-141.

Keil, H., Keil, S. \& Williams, P. A. (1987). Molecular analysis of regulatory and structural $x y l$ genes of the TOL plasmid pWW534. J Gen Microbiol 133, 1149-1158.

Kunz, D. A. \& Chapman, P. J. (1981). Catabolism of pseudocumene and 3-ethyltoluene by Pseudomonas putida (arvilla) $\mathrm{mt}-2-$ evidence for new functions of the TOL ( $p$ WWO) plasmid. J Bacteriol 146, 179-191.

Lebens, M. R. \& Williams, P. A. (1985). Complementation of deletion and insertion mutants of TOL plasmid pWWOregulatory implications and location of $x y l C$ gene. $J$ Gen Microbiol 131, 3261-3269.

Miller, V. L. \& Mekalanos, J. J. (1988). A novel suicide vector and its use in construction of insertion mutations - osmoregulation of outer-membrane proteins and virulence determinants in Vibrio cholerae requires toxR. J Bacteriol 170, 2575-2583.

Murray, K., Duggleby, C. J., Sala-Trepat, J. M. \& Williams, P. A. (1972). The metabolism of benzoate and methylbenzoates via the meta-cleavage pathway by Pseudomonas arvilla mt-2. Eur J Biochem 28, 301-310.

Polissi, A., Bestetti, G., Bertoni, G., Galli, E. \& Deho, G. (1990). Genetic analysis of chromosomal operons involved in degradation of aromatic hydrocarbons on Pseudomonas putida TMB. $J$ Bacteriol 172, 6355-6362.

Reid, M. F. \& Fewson, C. A. (1994). Molecular characterization of microbial alcohol dehydrogenases. Crit Rev Microbiol 20, 13-56.

Shaw, J.P. \& Harayama, S. (1990). Purification and characterization of TOL plasmid-encoded benzyl alcohol dehydrogenase and benzaldehyde dehydrogenase of Pseudomonas putida. Eur J Biochem 191, 705-714.
Shaw, J. P. \& Harayama, S. (1995). Characterization in vitro of the hydroxylase component of xylene monooxygenase, the first enzyme of the TOL plasmid-encoded pathway for the mineralization of toluene and xylenes. J Ferment Bioeng 79, 195-199.

Shaw, J. P., Rekik, M., Schwager, F. \& Harayama, S. (1993). Kinetic studies on benzyl alcohol dehydrogenase encoded by TOL plasmid pWWO - a member of the zinc-containing long-chain alcohol-dehydrogenase family. J Biol Chem 268, 10842-10850.

Suzuki, M., Hayakawa, T., Shaw, J. P., Rekik, M. \& Harayama, S. (1991). Primary structure of xylene monooxygenase - similarities to and differences from the alkane hydroxylation system. J Bacteriol 173, 1690-1695.

Taira, K., Hirose, J., Hayashida, S. \& Furukawa, K. (1992). Analysis of $b p h$ operon from the polychlorinated biphenyl-degrading strain of Pseudomonas pseudoalcaligenes KF707. J Biol Chem 267, $4844-4853$.

Vieira, J. \& Messing, J. (1982). The pUC plasmids, an m13mp7derived system for insertion mutagenesis and sequencing with synthetic universal primers. Gene 19, 259-268.

Wang, Y., Rawlings, M., Gibson, D. T., Labbe, D., Bergeron, H., Brousseau, R. \& Lau, P. C. K. (1995). Identification of a membrane protein and truncated LysR-type regulator associated with the toluene degradation pathway in Pseudomonas putida F1. Mol Gen Genet 246, 570-579.

Wheatcroft, R. \& Williams, P. A. (1981). Rapid methods for the study of both stable and unstable plasmids in Pseudomonas. $J$ Gen Microbiol 124, 433-437.

Williams, P. A. \& Murray, K. (1974). Metabolism of benzoate and the methylbenzoates by Pseudomonas arvilla mt-2: evidence for the

existence of a TOL plasmid. $J$ Bacteriol 120, 416-423.

Worsey, M. J. \& Williams, P. A. (1975). Metabolism of toluene and xylenes by Pseudomonas putida (arvilla) mt-2: evidence for a new function of the TOL plasmid. J Bacteriol 124, 7-13.

Received 8 March 1996; revised 11 July 1996; accepted 21 August 1996. 